\title{
Éléments pour une « archéologie » de la méthode directe
}

Henri Besse

\section{(2) OpenEdition \\ 12 Journals}

Édition électronique

URL : https://journals.openedition.org/dhfles/3386

DOI : 10.4000/dhfles.3386

ISSN : 2221-4038

Éditeur

Société Internationale pour l'Histoire du Français Langue Étrangère ou Seconde

Édition imprimée

Date de publication : 1 décembre 2012

Pagination : 11-30

ISSN : 0992-7654

Référence électronique

Henri Besse, "Éléments pour une « archéologie » de la méthode directe », Documents pour l'histoire du français langue étrangère ou seconde [En ligne], 49 | 2012, mis en ligne le 04 juillet 2016, consulté le 28 mai 2021. URL : http://journals.openedition.org/dhfles/3386 ; DOI : https://doi.org/10.4000/dhfles. 3386

Ce document a été généré automatiquement le 28 mai 2021.

(C) SIHFLES 


\title{
Éléments pour une " archéologie " de la méthode directe
}

\author{
Henri Besse
}

1 Marc Bloch se disait convaincu que « sans se pencher sur le présent, il est impossible de comprendre le passé »(1996: 30). La préoccupation présente d'une didactique du plurilinguisme et du pluriculturalisme ${ }^{1}$ permet de mieux comprendre ce qu'on pourrait appeler une " archéologie » de ce qui sera dénommé à la toute fin du XIX ${ }^{\mathrm{e}}$ siècle, après bien d'autres appellations, la méthode directe ${ }^{2}$.

2 Une de ses options fondatrices est, dans ses variantes orthodoxes, d'enseigner une L2 à des débutants sans que leur maître ait recours à leur L1, « directement » à la manière dont on apprend "naturellement» une L1. Option unilingue qui l'oppose au bilinguisme inhérent aux méthodes qui recourent à la traduction (L2-L1). Notons, toutefois, que cet unilinguisme est quasi inévitable quand le maître ne parle pas la L1 de ses élèves, ou quand il enseigne à des classes n'ayant aucune langue (leur L1 ou une première L2) en commun. Le maître ne pouvant faire autrement, il ne s'agit pas alors d'une véritable option méthodologique.

3 On sait qu'en Europe, jusqu'aux $\mathrm{XV}^{\mathrm{e}}-\mathrm{XVI}^{\mathrm{e}}$ siècles, seules les langues « savantes » (latin, grec et un peu d'hébreu) étaient dûment enseignées, les maîtres procédant, peu ou prou, comme ils les avaient apprises eux-mêmes. Les langues «vulgaires » (L1 ou L2), non encore outillées de grammaires et de dictionnaires, non encore "grammatisées ", s'apprenaient par le seul usage, plus oral qu'écrit, en interaction avec des natifs. Ce n'est que lorsque ces "vulgaires » furent " grammatisés », que se posa la question de savoir s'il valait mieux les enseigner à la manière des "savantes ", c'est-à-dire dans le cadre d'une approche au moins bilingue (L2-L1), ou selon une autre approche, plus ou moins imitée de l'apprentissage « naturel » d'une L1 ou d'une L2. 


\section{Apprendre une langue au seul moyen d'elle-même}

4 Rappelons brièvement deux exemples, dont le premier a été plus souvent repris que le second, attestant qu'enseigner une L2 sans avoir recours à la L1 de l'apprenant est une pratique bien antérieure à la méthode directe proprement dite.

\section{1 « L'expédient » du père de Michel de Montaigne}

5 Le compte rendu qu'en fait Montaigne à la fin du chapitre XXVI du Livre I de ses Essais (1927 : 206-254) est fréquemment cité mais pas assez analysé. Intitulé « De l'institution des enfans ${ }^{3}$ ", ce chapitre s'ouvre par une critique de l'éducation telle qu'elle était en son temps donnée dans les collèges, Montaigne proposant qu'on y débute par l'étude de deux langues « vulgaires » (« Ie voudrois premierement bien sçauoir ma langue, \& celle de mes voisins, où i'ay plus ordinaire commerce ", p. 247) avant de se mettre au latin et au grec (ibid.) $)^{4}$.

6 Persuadé que « la seule cause pourquoy nous ne pouuions arriuer à la grandur d'ame et de conoissance des anciens Grecs \& Romains » (ibid.) était les années qu'on perdait dans les collèges à mal apprendre leurs langues, son père imagina donc un " expedient » que Montaigne narre sur près de quatre pages (p. 247-251) que nous réduisons à ces quelques citations. L'infans Montaigne fut confié, «auant le premier desnouement de (s)a langue ", à un Allemand « du tout ignorant de nostre langue, \& tresbien versé en la Latine », lequel, aidé de " deus autres moindres en sçauoir pour me suiure», ne devait (et ne pouvait) l'entretenir qu'en latin. Il apprit ainsi à parler cette langue savante « sans art, sans liure, sans grammaire ou precepte, sans fouet \& sans larmes", alors qu'il ne parlait pas " plus de François ou de Perigordin que d'Arabesque ». Quant au grec, son père "desseigna me le faire apprendre par art, mais d'vne voie nouuelle par forme d'ébat \& d'exercice. Nous pelotions nos declinaisons à la manière de ceux qui, par certain ieux de tablier, apprennent l'Arithmetique \& le Geometrie». Si bien que lorsqu'il entra au collège de Guyenne ${ }^{5}$, entre six et sept ans, il maniait oralement mieux ces deux langues savantes que certains de ses maitres, mais, en raison de l'enseignement qu'il y reçut, il en « perdit incontinent » l'usage.

7 Cet «expedient" a été souvent rappelé du XVII ${ }^{\mathrm{e}}$ siècle $^{6}$ à nos jours. Mais, parmi d'autres, ni H. H. Stern (1983) ni C. Puren (1988) ne soulignent que Montaigne apprit son latin comme une sorte de L1 et non comme une L2. C. Germain (1993 : 73-74) le note, mais voit en Montaigne "l'un des défenseurs de l'apprentissage précoce des langues étrangères ", ce qui n'est pas exactement le cas (voir ci-dessus). Et l'on ne tient pas assez compte du contexte, très profondément différent du nôtre ${ }^{7}$, au sein duquel Montaigne a vécu et décrit cet « expedient ». Pas plus que l'on ne s'interroge sur ce qui fit que Montaigne désapprit «incontinent» son latin et son grec de la prime enfance quand il dut apprendre à les lire, à les écrire et à les raisonner grammaticalement, dans des variétés sans doute différentes de celles dont l'avait nourri son précepteur-nourrice et ses deux aides.

\section{2 « La belle économie de la maison de Robert Etienne »}

Durant les mêmes années où le jeune Montaigne apprenait à parler son latin, Robert Estienne $^{8}$ imposa à ceux qui travaillaient dans son imprimerie l'habitude d'y parler 
uniquement cette langue, son but étant d'améliorer la correction des ouvrages publiés dans celle-ci. Adrien Baillet ${ }^{9}$ avait décrit cette pratique dans son Jugement des Savants, et c'est à lui que se réfère ici $\mathrm{Ch}$. Rollin :

On sait, dit cet Auteur, la belle économie de la maison de Robert Etienne. Il ne recevoit dans son Imprimerie que des ouvriers habiles en grec \& en latin capables d'être maîtres ailleurs. Il avoit outre cela des valets \& des servantes, à qui il étoit défendu, aussi bien qu'à tous les ouvriers de l'Imprimerie, de parler autrement que latin. Sa femme \& sa fille l'entendoient fort bien, \& étoient de concert avec tous les domestiques pour ne point parler autrement. De sorte que les magazins, les chambres, la boutique, la cuisine, en un mot depuis le toit jusqu'à la cave tout parloit latin chez Robert Etienne (Rollin 1732, t. IV : 648).

Il s'agissait donc de faire apprendre le latin à des adultes ayant déjà en commun au moins une autre langue, la française dans l'une ou l'autre de ses variétés, par une sorte d'enseignement/apprentissage mutuel, lequel était pratiqué dans un milieu professionnel multilingue. D'autant plus que, toujours selon Rollin, «ce généreux Imprimeur avoit ordinairement chez lui dix hommes de lettres, tous des pays étrangers, faisant sous lui l'office de correcteurs des impressions" (ibid.). Cette pratique se retrouve tout au long de l'histoire de l'enseignement/apprentissage des L2, y compris de nos jours pour le latin ${ }^{10}$.

\section{3 À propos de l'enseignement du latin et du grec dans les collèges de l'époque}

Le trilinguisme (latin, grec et français hors de ses murs) pratiqué dans l'imprimerie Estienne, tout comme celui que pratiqua Montaigne au collège de Guyenne, n'était guère différent de celui qui domina dans les collèges et lycées de France au moins jusqu'au milieu du $\mathrm{XX}^{\mathrm{e}}$ siècle, et qui est encore au fondement de nos actuelles agrégations dites de grammaire et de lettres classiques ${ }^{11}$.

11 Ce que préconise la Ratio studiorum [1595] de la Compagnie de Jésus pour les trois " classes de grammaire ", c'est-à-dire pour des quasi ${ }^{12}$ débutants en latin et des vrais débutants en grec, permet de se faire une idée plus précise de ce qui avait cours dans la plupart des collèges du temps. On y enseignait certes le latin et le grec à l'opposé de ce qu'avait pratiqué le jeune Montaigne avec son précepteur-nourrice, «avec art, avec livre, avec grammaire ou préceptes » et parfois « avec fouet \& larmes ». Mais, dans le manuel de grammaire latine, les règles du latin étaient le plus souvent formulées « directement » en latin, pratique qui perdurera jusqu'au XVIII ${ }^{e}$ siècle ( $c f$. les rééditions des manuels de Despautère et d'Alvarez ${ }^{13}$ ). Ce que les prudentes modalisations de $\mathrm{Ch}$. Rollin, au tout début de son traité sur "l'étude de langue latine », confirment a contrario (1732, t. I : 160) : « Il me semble qu'à présent l'on convient assez généralement que les premieres régles que l'on donne pour apprendre le latin, doivent être en françois. » Et l'on ne peut exclure que le précepteur allemand du jeune Montaigne, avec qui il "pelotait " ses déclinaisons grecques à la manière des jeux de "tablier ", ou les ouvriers de l'imprimerie Estienne, du moins ceux qui étaient « habiles en grec \& en latin capables d'être maîtres ailleurs ", n'aient eu recours, à l'occasion, à cette forme très ancienne de l'exercice qu'est la chrie (chria). Exercice favori des rhéteurs mais dont les maîtres grammairiens usaient aussi, en faisant décliner à leurs élèves un paradigme grammatical sans avoir à l'expliciter en L1, par la simple répétition, plus ou moins dialoguée, d'une série d'exemples à même de l'illustrer. 
12 Bref, pendant des siècles, l'enseignement des préceptes grammaticaux ne semble pas avoir échappé à cette « règle commune » que la Ratio studiorum imposait aux régents des trois « classes de grammaire» :

Avant tout on conservera strictement l'usage de parler latin, sauf dans les classes où les élèves ne savent pas le latin; de sorte qu'il ne sera jamais permis, pour tout ce qui concerne la classe, d'utiliser la langue maternelle; on infligera aussi des blâmes à ceux qui n'en tiendraient pas compte; et à cette fin, le maître parlera continuellement latin (Ratio studiorum 1997 : 155).

13 Ce n'est qu'en troisième année que l'écrit, en L2 plus qu'en L1, était vraiment abordé par des exercices du type thèmes ou versions (ibid. : 182). Il est donc clair que, au moins pour les deux premières " classes de grammaire ", on était sans doute plus proche de ce qui pouvait se passer dans l'imprimerie de Robert Estienne, voire de ce qui se passera dans les classes suivant la méthode directe, que dans celles qui mettront en pratique, en particulier aux XIX ${ }^{e}$ et XX ${ }^{e}$ siècles, la méthode grammaire-traduction.

\section{De l'enseignement du latin et des langues « vivantes » au XVIIIe siècle}

$14 \mathrm{Au}$ cours de ce siècle se dégage un relatif consensus, au moins parmi les didacticiens ${ }^{14}$, quant à la nécessité de débuter l'enseignement des langues, tant pour les filles que pour les garçons, par celui de leur L1, qu'il s'agit d'apprendre scolairement " par principes ", ceux-ci pouvant dès lors être réutilisés pour enseigner le latin et le grec. Mais il se caractérise aussi par de vifs débats, parmi même ces spécialistes, entre ceux qui préconisent de débuter l'enseignement des $\mathrm{L} 2$ par les règles et ceux qui pensent qu'une certaine routine préalable à leur enseignement est nécessaire. Bornons-nous à rappeler quelques propositions, bien connues mais rarement analysées du point de vue qui est le nôtre ici, de deux partisans de cette seconde option : Dumarsais et Radonvilliers. Elles sont, par leur application dans les classes, éloignées de ce que sera la méthode directe (on y débute par une double traduction de textes " authentiques »), mais la théorisation qu'ils en font ne l'est guère de celles que proposeront les tenants de la méthode directe.

\subsection{Quand « la routine doit précéder les règles »}

15 Dumarsais, reconnaissant ce qu'il doit à « M. Locke dans son Traité de l'Education des Enfans ", résumait sa méthode à la formule : «la routine doit précéder les régles » (Dumarsais $1722: 2$ ). Ce qui, selon lui, allait à l'encontre des « Méthodes ordinaires » où l'on « aprend le Latin à peu près comme feroit un homme, qui pour aprendre à parler à un enfant, commenceroit par lui montrer la Mécanique des organes de la parole» (ibid.: 3). D'où une méthode qui se veut « une imitation raisonnée de la maniére dont on aprend les Langues vivantes » (ibid.: 1), «par usage » plus que "par règles». Pour une langue vivante, il faut « l'entendre parler à ceux à qui elle est naturelle, la parler avec eux, et s'éxercer ensuite à écrire dans cette langue »; pour les langues mortes, on y substitue ce qui s'en rapproche le plus, à savoir la lecture « d'ouvrages des anciens qui ont le mieux écrit en ces langues ", leur compréhension étant facilitée par une double traduction magistrale, la première interlinéaire et aussi littérale que possible, la seconde plus élégante. Et Dumarsais de refuser qu'on débutât par « la composition des thèmes », persuadé que « cette pratique est contraire à l'ordre naturel » $(1987: 37)$. 
Radonvilliers reconnaît quant à lui ce que sa méthode, qu'il dira de la «double version », doit à «M. du Marsais» (Radonvilliers 1768 : XII), au «sage Rollin » (ibid. : XIII), et à Locke. Tout comme Dumarsais, à première vue, le " problème » dont il part au début de son ouvrage semble éloigné de celui des tenants de la méthode directe: "Sachant une première langue, en apprendre une seconde par la lecture » (ibid. : XX, italiques originales). Mais la manière dont il le raisonne et le résout en est pourtant proche.

Son premier chapitre, qui couvre presque un quart de l'ouvrage, est en effet consacré à «l'étude de la langue maternelle » (ibid.: 1-54), par quoi il entend la manière dont on l'acquiert «naturellement ». Il y débute par une réflexion qu'il juge lui-même «assez bizarre » :

Il semble qu'un enfant devroit savoir la Langue de son pays, pour être en état de l'apprendre. On ne lui parle que le françois, pour lui enseigner le françois. Mais quelle utilité retirera-t-il des paroles qui frappent son oreille, si son esprit n'en connoît pas la valeur? Il faut donc qu'il ait reçu auparavant d'autres leçons. En effet, la Nature lui en a donné, tandis qu'il étoit encore au berceau : \& voici peutêtre la première. Lorsque l'enfant a senti de la douleur, ce sentiment lui a arraché des cris. Son âme réfléchissant sur elle-même, a combiné le sentiment de douleur avec les cris qui l'accompagnoient. Dès-lors il a su qu'un certain cri signifie la douleur. Sur ce fondement s'est élevé l'édifice d'une Langue entière, qu'on peut appeller la Langue naturelle (ibid. : 1-2).

18 Autrement dit, une L1 s'apprend certes au moyen d'elle-même, mais, pour être comprise de l'infans, celui-ci doit nécessairement disposer d'une autre "langue » à même de lui servir de truchement, une «Langue naturelle » non verbale (faite de cris, de gestes, de mimiques, de regards, de ressentis, de comportements, de situations et de réflexions vécues avec ses proches) qui l'aide à interpréter ce qui lui est dit dans ce qui n'est pas encore sa L1. "Langue » qui est, pour Radonvilliers, "la plus véridique " (ibid. : 18), « la plus pathétique » (ibid. : 19), qui « a cours d'un bout de la terre à l'autre » et qui « semble même » ne pas être "ignorée des animaux » (ibid.: 21). Une " différence essentielle » la distingue des langues articulées : «Le lien qui unit la pensée aux gestes est naturel \& nécessaire ; celui qui l'unit aux paroles est libre \& de convention » (ibid. : 17). Il s'ensuit qu'elle est à même de servir de truchement ou d'interprétant à n'importe quelle langue particulière.

19 Citons deux brefs passages où Radonvilliers décrit avec une grande finesse comment cette «Langue naturelle » aide l'infans à apprendre sa L1. Le premier est relatif « aux noms des objets qui ne tombent pas sous les sens » (p. 40-41):

Comment expliquer par des gestes ce que signifie le terme reconnoissance? Un hasard dont la gouvernante saura profiter, lui en fournira le moyen. L'enfant desire vivement quelque chose; il l'obtient. S'il est né reconnoissant, son cœur sera pénétré en ce moment d'un sentiment inconnu. La gouvernante prononce alors le mot reconnoissance; \& par un geste elle l'applique à cette émotion nouvelle : la liaison entre le sentiment \& le mot commence à se former, \& dans la suite elle se resserrera par la répétition du même événement (Radonvilliers $1768: 40-41$ ).

Le second concerne « des inflexions \& des particules» :

La Langue naturelle ne connoit point les mots de ces deux classes, ainsi elle ne peut pas substituer des équivalents de la même espèce. La totalité d'une action extérieure y supplée. L'enfant sollicite des yeux \& de la main la permission de jouer, la gouvernante lui répond des yeux \& de la main, c'est-à-dire, dans leur Langue commune entre'eux, qu'elle le lui permet, en même temps elle dit, jouez; l'action extérieure interprète l'inflexion jouez. Une autre fois l'enfant veut jouer avec la gouvernante, elle le refuse ; \& lui faisant signe d'aller trouver son frère, elle dit en 
même temps, jouez avec votre frère. Il comprend par-là le sens de la particule avec

(Radonvilliers $1768: 42$ ). méthode connue, [...] pourquoi chercher une autre méthode pour lui apprendre une seconde Langue? Si l'art suit exactement les procédés de la nature, il aura le même succès. » D'où sa méthode " par une double version, l'une des mots, l'autre de la pensée » qu'il applique d'abord au latin qui ne se peut enseigner par la conversation, ce qui « seroit sans contredit le moyen le plus naturel \& le plus sûr ", " puisqu'on ne parle plus cette Langue » (ibid. : 65). Il y substitue donc, tout comme Dumarsais, « la lecture, qui en approche beaucoup » (ibid.). Ce qui «n'exige d'un commençant aucun travail qui puisse être au dessus de ses forces, nul principe à comprendre, nulle règle à appliquer, nul raisonnement à faire » (ibid. : 90). Avant d'en faire pourtant l'application, dans son dernier chapitre (ibid. : 236-268), à des langues qui se parlent, à l'allemand, l'anglais, l'espagnol et l'italien.

\subsection{La pédagogie des « choses qui se puissent montrer au doit » de l'abbé Pluche et la méthode « par l'intuition sensible » de Basedow}

23 Si proches que soient les raisonnements didactiques de Dumarsais et de Radonvilliers de ceux qui sous-tendront, deux siècles plus tard, la méthode directe, il n'en reste pas moins qu'ils débutent leur enseignement d'une L2 autrement que cette dernière. Mais d'autres didacticiens du XVIII ${ }^{\mathrm{e}}$ siècle s'en rapprochent bien davantage, sans qu'on puisse parler, à leur propos, de précurseurs de la méthode directe ${ }^{16}$. Deux exemples, là aussi bien connus des spécialistes mais qui sont « lus », en général, autrement que nous le faisons ici.

Le premier est celui de N.-A. Pluche, dit souvent l'abbé Pluche, dans La mécanique des langues, et l'art de les enseigner. Il y débute son « Livre second » ainsi :

Il ne peut y avoir que deux façons d'apprendre les langues. [...] Si vous commencez l'apprentissage d'une langue par la pratique, c'est-à-dire, par la très-constante habitude d'entendre puis de répéter des choses bien dites, \& qu'ensuite vous affermissiez cet usage par l'étude des régles, par la connoissance de la Grammaire ; voilà le vrai chemin. [...] Si vous commencez l'apprentissage par le raisonnement, si sans aucun usage préallable de la langue, vous prétendez sous la direction d'une Grammaire passer d'une première régle à celles qui viennent ensuite à la file [...]; cette route est bien longue : elle vous assujettit à de longs détours (Pluche $1751: 40$, italiques originales).

Ce qui singularise Pluche en son siècle, c'est l'attention qu'il porte à l'oralité des langues, qu'elles soient vivantes ou mortes. «Tel est l'ordre de la nature que la langue 
de l'homme soit sous le gouvernement de l'oreille» (ibid.: 67, en petites majuscules dans le texte original). Ce que notre abbé argumente en s'appuyant (ibid.: 68) sur l'exemple des sourds-muets ${ }^{17}$, qui sont muets parce que sourds (« celui qui vient au monde sans l'usage de l'ouïe, se trouve nécessairement privé de celui de la parole »), et sur l'exemple de ce qu'on appellera plus tard des enfants-loups. D'où un "premier degré de l'apprentissage des langues ", qui apparait comme très proche de celui de la méthode directe :

Entretenez la jeunesse de choses qui se puissent montrer au doit. Si vous ne les avez point sous vos yeux, faites concourir plusieurs signes précédemment connus qui les rendent comme présentes. Si les objets même ou la description qu'on peut en faire se trouvent agréables, les signes que vous employez pour en parler se collent pour ainsi dire à l'objet, \& tiennent individuellement les uns aux autres. C'est l'action de la nature même (Pluche $1751: 140-141$, nous soulignons).

Ce qu'il glose, peu après, en s'appuyant sur l'acquisition « naturelle » non plus d'une L1 comme Radonvilliers mais d'une L2 :

C'est ici qu'il faut faire ce que nous avons remarqué qui se pratiquoit pour un Étranger qui arrive parmi nous. On lui montre exactement chaque objet dont il faut lui parler. A la vûe ou à l'indication suffisante des choses, on ajoute aussitôt les termes qui les expriment, \& on donne à ces termes leur juste assemblage. [...]. Le commencement se réduit là. Les choses \& les signes entreront de compagnie dans la mémoire (Pluche $1751: 144$ ).

Et comme «la vûe \& l'accès de bien des choses ne sont pas toujours à notre disposition ", Pluche préconise d'avoir alors recours à des images :

Rien n'est ici plus parfaitement d'accord avec les inclinations de l'homme \& avec l'expérience universelle que de présenter aux jeunes gens de bonnes estampes de la plupart des choses à la portée desquelles il n'est pas communément possible de les mettre. [...] Il ne faut que chiffrer chaque partie dans l'estampe \& transporter sur une autre feuille les mêmes chiffres accompagnés des noms propres qui y conviennent. Dès que vous mettrez le doit sur un point de l'estampe, on vous en livrera le nom ; \& si vous nommez vous-même la partie qu'il vous plait de choisir dans une grande figure ; l'enfant portera aussitôt le doit sur le point que vous avez nommé (ibid. : 150-151).

Ce ne sont certes pas exactement les termes dont useront les tenants de la méthode directe, mais l'idée sous-jacente est voisine : il s'agit d'associer aussi «directement » que possible ce qui est perçu par les yeux de l'élève à ce qu'il perçoit par l'oreille de la L2 qu'il commence à apprendre.

Notre second exemple est emprunté aux travaux de Marcus Reinfried, dont nous reprendrons succinctement ici un seul article (1990). Il porte sur l'enseignement des L2 (en particulier du latin et du français) tel qu'il fut mis en œuvre dans l'internat, dénommé Philanthropinum, que Johann Bernhard Basedow (1724-1790) avait ouvert à Dessau en 1774, établissement qu'il quitta dès 1778 et qui fut définitivement fermé en 1793. Auteur d'une thèse de Magister sur l'apprentissage "naturel », il avait publié, l'année même de l'ouverture de cet établissement, un manuel destiné aux enfants (Das Elementarwerk $\left.{ }^{18}\right)$. Établissement où il fut rejoint par des disciples de vingt à trente ans plus jeunes que lui, dont «les deux Français de Dessau» (Chalmel 2004 : 143), JeanFrédéric Simon (1747-1829) et Jean Schweighaüser (1753-1801), l'un et l'autre d'origine strasbourgeoise et au moins trilingues (allemand-français-latin).

Basedow avait lu l'Émile [1762] de Jean-Jacques Rousseau, mais celui-ci doutait que « nul enfant, les prodiges à part, ait jamais vraiment appris deux langues ", et rangeait leur 
enseignement « au nombre des inutilités de l'éducation $»^{19}$. Basedow ne put donc guère s'en inspirer pour ce qui est de l'enseignement des L2. Avait-il lu les ouvrages de Radonvilliers ou de l'abbé Pluche dont il vient d'être question? Toujours est-il que ce qu'on pratiqua à Dessau, une méthode dite "par l'intuition sensible " (Versinnlichende Sprachlehrmethode), ne différait guère de ce que ces deux didacticiens français avaient préconisé quelques années plus tôt. Un de ses disciples, Christian Heinrich Wolke, la mit en œuvre en allant avec ses élèves se promener autour de Dessau avec eux, leur nommant en français ou en latin (L2) tout ce qu'ils voyaient, entendaient, sentaient, goûtaient ou éprouvaient, ou bien, pour les plus jeunes, au moyen de divers jeux ${ }^{20}$ menés dans l'une ou l'autre de ces L2. Selon Reinfried :

Pour la première fois dans l'enseignement des langues étrangères en Allemagne, l'image était devenue un intermédiaire systématiquement utilisé pour l'accès direct au sens des mots ou des phrases. L'emploi des images devait selon [...] Basedow [...] rendre possible le monolinguisme, surtout au cours des deux premières années de l'enseignement du français. [...] Des explications dans la langue maternelle ne (devaient) être données que dans « des cas exceptionnellement rares » (Reinfried $1990: 135-136)$.

31 Wolke serait, selon Reinfried, « le seul parmi les philanthropistes » (ibid.) à avoir justifié didactiquement cette option. Pour lui, elle incite les apprenants, privés de la facilité que leur donne une traduction, à faire l'effort de deviner le sens des sons "étrangers ", et partant, les incite à concentrer leur attention sur ceux-ci et sur leur mémorisation. Argument qu'on retrouvera, au XXe siècle, chez certains tenants de la méthode directe ou de la méthode SGAV. Elle permet aussi « que l'expression dans la langue étrangère se lie à la représentation de l'objet référentiel en formant une unité organique » (ibid.), ce qui était déjà l'argument principal de Pluche. Il sera théorisé par certains tenants de la méthode directe (Reinfried cite, à ce propos, "un petit livre de Félix Franke », daté de 1884) à partir de la psychologie "associationniste » de leur temps. Le Philanthropinum est célèbre par une démonstration publique de sa méthode à laquelle "assistèrent des hommes de lettres, des ecclésiastiques et des pédagogues de toutes les parties de l'Allemagne » (ibid. : 130), démonstration qui fut assurée par l'un des « deux Français de Dessau », H. H. Simon, lequel étant au moins bilingue allemand-français, la fit donc non par nécessité mais bien par méthode.

\section{Des techniques quasi oubliées que la méthode directe reprendra à la fin du $\mathrm{XIX}^{\mathrm{e}}$ siècle}

Deux constats s'imposent après examen de ces quelques rappels (parmi d'autres possibles) bien antérieurs à l'émergence de la méthode directe.

\subsection{De la relative permanence des techniques d'enseignement/ apprentissage des L2}

Le premier est que les techniques qui la caractérisent habituellement - en particulier, celles relatives à l'accès « direct » au sens des signes de la $\mathrm{L} 2$, ou celles relatives à une certaine routine orale préalable à l'enseignement de ses règles grammaticales -, ont été pratiquées et préconisées bien avant qu'elles ne le soient par les tenants de la méthode directe, lesquels ignoraient volontiers ceux qui les avaient précédés dans cette voie. Le second est que le courant dans lequel elle s'inscrit - qu'on pourrait dire, puisqu'il vise à 
imiter la manière dont on acquiert «naturellement» une langue (L1 ou L2), «naturaliste » - s'est, durant des siècles, inscrit dans un plurilinguisme combinant langues « savantes » et langues « vulgaires ». L'unilinguisme de ces techniques n'y était que d'ordre méthodologique, en ce qu'il ne s'appliquait qu'aux débutants et qu'il n'excluait pas des activités mettant en jeu d'autres langues, dont la L1 des élèves. L'idée de faire appel à des natifs de la L2 ne maîtrisant pas la L1 de leurs élèves ${ }^{21}$ y est quasi absente. En d'autres termes, les didacticiens de ce courant raisonnaient dans le cadre d'une didactique des langues plus que dans celui de la didactique d'une langue, ce qu'auront tendance à faire ceux de la méthode directe.

À preuve cette phrase d'une instruction officielle française du 13 novembre 1890 que Christian Puren juge être «le principe fondateur de la DLVE ${ }^{22}$ comme discipline autonome » (1988: 85-86, en gras dans son texte) : "Une langue s'apprend par ellemême et pour elle-même, et c'est dans la langue, prise en elle-même, qu'il faut chercher les règles de la méthode ". Point de vue clairement unilingue, en parfaite conformité avec l'idéologie nationaliste de l'époque, qui fait de chaque langue une réalité non seulement différente des autres langues mais quasi indépendante de cellesci, réalité dont l'organisation interne devrait à elle seule déterminer l'art de l'enseigner. L'on aura, dès lors, autant de didactiques différentes que de langues enseignées, et l'état présent des institutions de formation des enseignants de L2, particulièrement en France, nous paraît toujours relever de cette idéologie monolingue (voir Besse 1993).

D'où la question de savoir comment l'on est passé, entre la fin du XVIII ${ }^{\mathrm{e}}$ et la fin du XIX siècle, d'une didactique des langues ouverte au plurilinguisme à cette juxtaposition de didactiques plus ou moins monolingues, à la fois selon la langue enseignée, et selon qu'elle l'est en tant que L1 ou en tant que L2 (telles les filières dites en France FLM et FLE). La réponse est à chercher, du moins c'est notre hypothèse, non dans l'histoire interne des méthodes mais dans leur histoire externe, celle qui est plus ou moins directement liée à leur émergence, à leur maintien et à leur déclin.

\subsection{Du multilinguisme inhérent aux royaumes et empires d'Europe au monolinguisme institué par ses États-nations}

Divers facteurs ont joué, mais ils nous paraissent tous surdéterminés par la complexe évolution des relations qui, en Europe au moins, ont été instituées, au cours de ces cinq derniers siècles, entre le politique, le religieux et les langues.

D'un point de vue rétrospectif, cette évolution peut se résumer au passage d'un principe, qui resta dominant jusqu'au traité de Vienne en 1815, de division des principautés, royaumes ou empires d'ordre politico-religieux à un principe d'ordre politico-linguistique, qui s'imposera peu à peu au cours du XIX siècle et qui est au fondement de la grande majorité de nos États-nations actuels. D'où une lente et complexe modification des représentations et des institutions relatives à ces trois domaines où l'on est passé d'un multilinguisme de fait - très rares sont encore de nos jours les communautés instituées en États à être strictement monolingues - à un monolinguisme davantage proclamé qu'effectif, auquel tous les ressortissants d'un même État sont censés s'identifier (Besse 1987). Très succinctement, on pourrait dire qu'on est passé de la maxime "tel prince, telle religion " (Cujus regio, ejus religio ${ }^{23}$ ), dominante en Europe de la fin du XVe au XVIII e siècles, à des maximes du type, pour les 
États constitués, «tel État, telle langue», et pour les communautés désirant se constituer en État, « telle langue, tel État », maximes qui l'ont emporté au cours du XIX ${ }^{\mathrm{e}}$ siècle dans presque toute Europe. Désormais, "l'aspiration des peuples à disposer d'eux-mêmes » et le «droit des nationalités » apparaîtront comme une sorte de norme européenne que nombre d'États éviteront cependant d'appliquer tant à leurs minorités linguistiques que dans leurs colonies, avec les conséquences que l'on sait jusqu'à nos jours.

De cette idéologie nationaliste, est née une juxtaposition de monolinguismes plus officiels que réels. D. Baggioni (1997) parle, à juste titre, d'un « processus de dallage de l'Europe en États-Nations ", chaque État se devant d'avoir, tout comme son drapeau, une langue pour ainsi dire identificatoire. Lourd "dallage» qui n'est pas, heureusement, sans défauts et quelques herbes folles. Il n'est parvenu, en près de deux siècles, ni à masquer le multilinguisme effectif de tous les États européens actuels, ni à éradiquer le plurilinguisme, plus répandu qu'on ne le dit souvent, de nombre de leurs ressortissants.

\subsection{Des conséquences de cette idéologie monolingue sur l'enseignement des langues}

Cette idéologie monolingue a joué un grand rôle dans la généralisation, en Europe, de l'enseignement primaire au cours du XIX siècle, tous les ressortissants d'un État devant, quelle que soit leur réelle L1, savoir parler, lire et écrire la langue " nationale ». D'où le succès, en France, de ce que l'inspecteur général de l'enseignement primaire, Irénée Carré, appelait, à la fin des années 1880 , la «méthode maternelle ${ }^{24}$. Proche dans ses raisonnements de ceux développés par les tenants de la méthode directe, il s'en distingue par une finalité d'ordre civilisatrice ou assimilatrice qui le conduira à écrire : «Ce sont des Français qu'il faut pour franciser les Bretons » (Carré $1888: 223$ ). Raisonnement qui l'emportera, à la même époque, dans les colonies françaises, les condamnant à la méthode directe par nécessité plus que par méthode (les instituteurs, essentiellement des « Français de France », ignoraient très généralement les L1 de leurs élèves).

L'enseignement secondaire resta plus ou moins à l'écart de cette idéologie monolingue, ayant été longtemps réservé aux enfants d'une classe minoritaire mais privilégiée au moins culturellement. Néanmoins, on y eut de plus en plus tendance, comme dans le primaire, à y recruter, par examen ou concours, des enseignants « nationaux » pour y enseigner les L2 vivantes. Ce qui eut pour effet d'écarter de cet enseignement leurs simples natifs qui l'assuraient jusqu'alors à moindre coût. Reinfried y voit une des causes du quasi oubli, en Allemagne durant le XIX siècle, de la méthode des philanthropinistes (1990: 143). Et l'on comprend que nombre de ces enseignants, non natifs de la langue qu'ils enseignaient en tant que L2, aient été des adeptes de la méthode grammaire-traduction plus que de la méthode directe.

41 Ces quelques éléments suggèrent, à défaut de démontrer, que les techniques les plus caractéristiques de la méthode directe ont été mises en pratique, décrites voire théorisées des siècles avant que ses tenants ne la promeuvent, vers 1900, en tant que telle. Et il serait aisé de montrer qu'il en va de même pour la méthode grammairetraduction qu'on lui oppose traditionnellement. En d'autres termes, ces éléments « archéologiques " questionnent une histoire de la didactique des langues (L1 et L2) 
réduite à une succession de méthodes, tant il est clair que l'émergence, le maintien et l'abandon de ces méthodes apparaissent davantage liés à l'histoire externe de cette didactique qu'à son histoire interne.

Ils attestent aussi qu'enseigner une L2 à des débutants au seul moyen d'elle-même a longtemps été compatible avec un certain plurilinguisme. L'unilinguisme de cette technique, qui revient à privilégier les natifs de cette L2 pour l'enseigner, peut donc l'être tout autant, pour peu qu'il soit extrait de l'idéologie monolingue qui lui a permis triompher durant quelques décennies. Ce qui implique de l'inscrire dans le cadre d'une didactique des langues qui soit autre chose qu'une juxtaposition ou comparaison des didactiques propres à chaque langue enseignée.

\section{BIBLIOGRAPHIE}

Sources

CARRÉ, Irénée (1888). « De la manière d'enseigner les premiers éléments du français dans les écoles de la Basse-Bretagne ». Revue Pédagogique, t. XII, 217-236.

DUMARSAIS, Charles Chesneau (1722). Exposition d'une méthode raisonnée pour apprendre la langue latine. Suivie de Le Poëme séculaire d'Horace. Paris : E. Ganeau.

DUMARSAIS, Charles Chesneau (1987) [1729-1756]. Les véritables principes de la grammaire et autres textes 1729-1756. Paris : Fayard.

MONTAIGNE, Michel de (1927) [1588]. Essais de Messire Michel de Montaigne publiés sur l'exemplaire de Bordeaux avec une introduction par Fortunat Strowski. Paris : Aux Éditions de la chronique des Lettres de France.

PLUCHE, Noël-Antoine (1751). La mécanique des langues, et l'art de les enseigner. Paris : Chez la Veuve Estienne \& Fils.

Ratio studiorum (1997) [1599]. Plan raisonné et institution des études dans la Compagnie de Jésus. Édition bilingue latin-français. Présentée par A. Desmoustier et D. Julia, traduite par L. Albrieux et D. Pralon-Julia, annotée et commentée par M.-M. Compère. Paris : Belin.

RADONVILLIERS, Claude-François Lyzarde de (1768). De la manière d'apprendre les langues. Paris : Chez Saillant.

ROLLIN, Charles (1732) [1726-1729]. De la maniere d'enseigner et d'etudier les belles lettres, Par raport à l'esprit \& au cœur. 4 t. Quatrième Édition, revue \& corrigée, \& augmentée d'une Table des matières. Paris : chez Jacques Estienne.

Études

BESSE, Henri (1993). « Pour une formation bivalente des enseignants de langues vivantes ». Les Langues Modernes, $\mathrm{n}^{\circ}$ 4. Paris : APLV (Association des Professeurs de Langues Vivantes), 45-55.

BIAGGIONI, Daniel (1997). Langues et nations en Europe. Paris : Payot.

BLOCH, Marc (1996) [1946]. L'étrange défaite. Paris : Gallimard. 
CHALMEL, Louis (2004). Réseaux philanthropinistes et pédagogie au XVIII ${ }^{e}$ siècle. Berne : Peter Lang.

GERMAIN, Claude (1993). Évolution de l'enseignement des langues : 5000 ans d'histoire. Paris : CLE International.

HOWATT, Anthony Philip Reid \& WIDDOWSON, Henry G. (2004). A History of English Language Teaching. $2^{\mathrm{e}}$ éd. Oxford : Oxford University Press.

PUREN, Christian (1988). Histoire des méthodologies de l'enseignement des langues. Paris : Nathan-CLE International.

REINFRIED, Marcus (1990). « Les origines de la méthode directe en Allemagne ». Documents pour l'histoire du français langue étrangère ou seconde, 6, 126-156.

STERN, Hans Heinrich (1983). Fundamental Concepts of Language Teaching. Oxford : Oxfor

\section{NOTES}

1. Cet article est la réduction d'une communication faite à l'université François Rabelais de Tours en mai 2012, qui portait sur l'incompatibilité présumée entre ce plurilinguisme-culturalisme et la méthode directe.

2. D'après C. Puren (1988: 94), le syntagme serait apparu, en France, pour la première fois dans une circulaire du 15 novembre 1901. Il se répand en Italie, en Belgique, en Espagne durant les mêmes années. Dans les pays germaniques et anglo-saxons, on préféra un temps parler de neusprachliche Reformbewegung ou de Reform movement, avant d'adopter, au début du XX $\mathrm{X}^{\mathrm{e}}$ siècle, des calques allemand (direkte Methode) et anglais (direct method) de méthode directe (voir A. P. R. Howatt \& H. Widdowson 2004 : 226-227).

3. Nous le citons, dans la typographie l'édition Strowski, sauf pour les $f$ imprimés avec barre à gauche que nous rendons par $s$. Les italiques signalent les corrections et additions manuscrites portées par Montaigne sur son texte de 1588.

4. Ce que préconisera aussi J. A. Comenius un demi-siècle plus tard.

5. Fondé en 1533 par la jurande de Bordeaux, ce collège n'était pas un collège jésuite, contrairement à ce qu'on lit parfois. Il fut d'ailleurs rapidement concurrencé par celui de la Magdeleine fondé par les jésuites dès 1587.

6. John Locke lui consacrera une quinzaine de pages dans Some Thoughts Concerning Education (1693).

7. Pierre Eyquem l'a voulu pour son fils dans un projet d'ascension sociale et nobiliaire.

8. Robert (1503-1559), plurilingue (français, latin, grec et hébreu), éditeur de textes dans ces langues et auteur du premier dictionnaire français-latin.

9. Adrien Baillet (1649-1706) fut le premier biographe de R. Descartes.

10. Que l'on pense au projet évoqué par N. Beauzée dans l'Encyclopédie (art. Méthode, 447) : ce «que l'on proposa dans le siecle dernier, \& que M. de Maupertuis a réchauffée de nos jours, de fonder une ville dont tous les habitans, hommes \& femmes, magistrats \& artisans ne parleroient que la langue latine ", ou à ce qui se fait actuellement en Belgique (avec l'école internationale Schola Nova fondée en 1995, où le latin est utilisé comme langue européenne de communication), en Italie (avec son Accademia Viviarum Novum qui propose, à Naples ou à Rome, des séjours durant lesquels les étudiants sont invités à ne parler que le latin et le grec ancien).

11. Celle de grammaire était originellement (à la fin du XVIII ${ }^{\mathrm{e}}$ siècle) destinée à recruter les enseignants des "classes de grammaire ", que suivaient tous les élèves entrant au collège ; celle de lettres qu'on dira plus tard de lettres classiques, aux enseignants des classes d'humanités et de rhétorique. 
12. Les élèves y avaient été familiarisés par la fréquentation de la messe en latin et par les prières quotidiennes (entre autres, le Pater noster et l'Ave Maria), à partir desquelles ils apprenaient le plus souvent à lire et à écrire.

13. L'édition originale de celui de Jean Despautère (Commentarii grammatici, 1509-1519), à la fois pour les élèves et pour les maîtres, ne contient que quelques rares traductions en français ou en néerlandais (L1 de ses utilisateurs potentiels). Celui de Manuel Alvares (De Institutione Grammatica Libri Tres, 1572), qui a connu plus de cinq cents éditions en Europe et hors d'Europe jusqu'au XIX ${ }^{\mathrm{e}}$ siècle, versifient en partie les règles du latin, et ne contient, originellement, aucune traduction L2-L1.

14. Le terme est utilisé en tant que substantif, à peu près dans le sens actuel, dès la fin XVIII ${ }^{\mathrm{e}}$ siècle.

15. C'est ainsi que Beauzée définit ce terme au début de son article « Méthode » de l'Encyclopédie

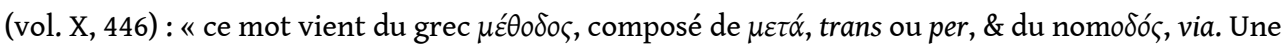
méthode est donc la manière d'arriver à un but par la voie la plus convenable ».

16. Ils ne sauraient l'être, au moins au sens premier et prophétique de ce terme, ignorants qu'ils étaient du futur au sein duquel cette méthode a fini par émerger.

17. Rappelons que P. Guberina, un des concepteurs de la méthode S.G.A.V., raisonnera de même.

18. J. B. Basedow : Das Elementarwerk. Ein Vorrath der besten Erkenntnisse zum Lernen, lehren. Dessau : Wiederholen und Nachdenken, 1774, en 4 tomes. La même année, il paraît (à Dessau et Berlin) dans une traduction en français.

19. J.-J. Rousseau : Émile ou de l'éducation [1762]. Paris : Librairie Garnier Frères, 1939, 105.

20. M. Reinfried (1990: 146) cite, d'après Wolke, le « jeu du commandement » (pour enseigner l'impératif, le maître dit aux élèves «Fermez les yeux !» ou « Frappez les mains ! ", en faisant ce qu'il dit, avant que les élèves ne le fassent d'eux-mêmes), le «jeu du cache-cache » pour enseigner les adverbes de lieu (il cache un objet que les élèves recherchent guidés par le maitre leur parlant en L2), ou le " jeu du dessin à deviner » (le maître l'esquisse, les élèves essaient de dire en $\mathrm{L} 2$ ce que sera la réalité dessinée ; le maître le complète, les élèves essaient de nouveau ; etc.).

21. C'est l'origine que l'on prête parfois à la « méthode Berlitz».

22. Didactique des Langues Vivantes Étrangères.

23. Elle apparaît dans un traité de droit canonique (Institutiones juris canoni) publié en Allemagne en 1612, dont l'auteur est un certain Joachim Stephani, mais fut mise ne pratique peu après la chute de Cordoue en 1592.

24. Carré, à notre connaissance, ne l'a jamais dénommée « directe ».

\section{RÉSUMÉS}

Les techniques d'enseignement les plus caractéristiques de la méthode directe ont été pratiquées et préconisées bien avant que cette méthode n'émerge en tant que telle, tout comme elles ont été longtemps compatibles avec un certain plurilinguisme. Elles peuvent le redevenir, pour peu qu'on les extraie de l'idéologie nationaliste qui a permis à cette méthode de triompher durant quelques décennies. Il s'ensuit qu'on ne peut s'en tenir à l'histoire de la didactique de la seule langue enseignée, pas plus qu'on ne peut réduire l'histoire de l'enseignement/apprentissage les langues (L1 ou L2) à la succession des méthodes par lesquelles elles l'ont été. 
The Direct Method's most characteristic teaching techniques were advocated and put into use long before the method appeared as such, just as much as, likewise, they were, for a long time, compatible with a certain form of multilingualism. This could happen again provided they are stripped off the nationalist ideology thanks to which they prevailed for few decades. It follows that one cannot simply take into account the history of the language taught, just as much as the history of the learning/teaching of (L1 or L2) languages cannot be restricted to the successive methods used for the purpose.

\section{INDEX}

Mots-clés : méthode directe, techniques d'enseignement des L2, monolinguisme, plurilinguisme, archéologie

Keywords : direct method, teaching technique, monolingualism, multilingualism, archaeology

\section{AUTEUR}

\section{HENRI BESSE}

ENS de Lyon

hebesse@laposte.net 